\title{
Fragen zum Thema „Ernährungsmodifikation und Stressmanagement“
}

\section{-1. Mit welcher Blutdrucksenkung ist statis- tisch betrachtet pro Kilogramm Gewichtsver- lust zu rechnen?}

A $-2,5 \mathrm{mmHg}$ systolisch, $-1,5 \mathrm{mmHg}$ diastolisch

B $-1,05 \mathrm{mmHg}$ systolisch, $-0,92 \mathrm{mmHg}$ diastolisch

C $-0,5 \mathrm{mmHg}$ systolisch, $-0,2 \mathrm{mmHg}$ diastolisch

D -0,1 mmHg systolisch, $-0,1 \mathrm{mmHg}$ diastolisch

E $-0,3 \mathrm{mmHg}$ syst., $-0,3 \mathrm{mmHg}$ diast.

\section{- 2. Welches Ziel der Kochsalzzufuhr ist} anzustreben?

A Unter $10 \mathrm{~g} / \mathrm{Tag}$

B Unter $8 \mathrm{~g} / \mathrm{Tag}$

C Unter $5 \mathrm{~g} / \mathrm{Tag}$

D Unter $3 \mathrm{~g} / \mathrm{Tag}$

E Unter 1,5 g/Tag

\section{- 3. Welche Maßnahme spielt neben der} Gewichtsabnahme für die diätetische Blutdruckbehandlung die wichtigste Rolle?

A Kochsalzrestriktion

B Verminderung der Eiweißzufuhr

C Reichliche Flüssigkeitszufuhr

D Hohe Zufuhr tierischer Fette

E Kohlenhydratrestriktion

- 4. Welche diätetische Maßnahme hat den geringsten Einfluss auf den Blutdruck?

\author{
A Vegetarische Ernährung \\ B Hohe Zufuhr mehrfach ungesättigter \\ Fettsäuren \\ C Reduktion des Alkoholkonsums \\ D Kochsalzarme Kost \\ E Weglassen von Kaffee
}
- 5. Die empfohlene Alkoholmenge pro Tag bei Patienten mit Hypertonie beträgt
A unter $10 \mathrm{~g}$.
B unter $20 \mathrm{~g}$.
C unter $25 \mathrm{~g}$.
D unter $50 \mathrm{~g}$.
E unter $100 \mathrm{~g}$.

- 6. Wie kann der Kochsalzkonsum, neben dem Weglassen des Salzstreuers, am besten eingeschränkt werden?

A Sich hauptsächlich von Konserven ernähren

B Möglichst viele Speisen selbst frisch zubereiten

C Sich hauptsächlich von Tiefkühlkost ernähren

D In die Kantine zum Essen gehen

E Häufig im Lokal essen

- 7. Wie viel g Alkohol enthält ein Liter Bier mit einer Alkoholkonzentration von 5 Volumenprozent?

A $50 \mathrm{~g}$

B $100 \mathrm{~g}$

C $5 \mathrm{~g}$
D $40 \mathrm{~g}$

E $20 \mathrm{~g}$

- 8. Wie hoch ist der durchschnittlich zu erwartende blutdrucksenkende Effekt (systolisch/ diastolisch) bei einer Umstellung auf vegetarische Kostform?

A $2-3 / 1-2 \mathrm{mmHg}$

B $3 / 2 \mathrm{mmHg}$

C $5-9 / 3-5 \mathrm{mmHg}$

D $10-12 / 8-10 \mathrm{mmHg}$

E $15 / 10 \mathrm{mmHg}$

- 9. Welche Kombination führt zur stärksten Blutdrucksenkung?

A Gewichtsabnahme + erhöhte Ballaststoffzufuhr

B Salzarme Kost (3 g/Tag) + Alkoholzufuhr von maximal $25 \mathrm{~g} / \mathrm{Tag}$

C Erhöhte Ballaststoffzufuhr + Kaffeeverzicht

D Vegetarische Lebensweise + erhöhte Zufuhr mehrfach ungesättigter Fettsäuren

E Gewichtsabnahme + salzarme Kost (3 g/Tag)

- 10. Mit welcher Blutdrucksenkung ohne Medikamente können Hypertoniker rechnen, die sich dem multidisziplinären Interventionsprogramm M.O.B.I.L.I.S. unterziehen?

A $-11 /-6 \mathrm{mmHg}$

B $-8 /-4 \mathrm{mmHg}$

C $-6 /-3 \mathrm{mmHg}$

D $-4 /-2 \mathrm{mmHg}$

E $-2 /-1 \mathrm{mmHg}$

\section{ANTWORTFORMULAR}

Ich versichere, alle Fragen ohne fremde Hilfe beantwortet zu haben.

Mit dem Einreichen dieses Fragebogens erklärt sich der Einreichende damit einverstanden, dass die angegebenen Daten zum Zweck der Teilnahmebestätigung gespeichert und bei erfolgreicher Teilnahme auch an den Einheitlichen Informationsverteiler (EIV) der Ärztekammern weitergegeben werden.
„Ernährungsmodifikation und Stressmanagement“ MMW-Fortschr. Med. Nr. 47 / 2006 (148. Jg.)

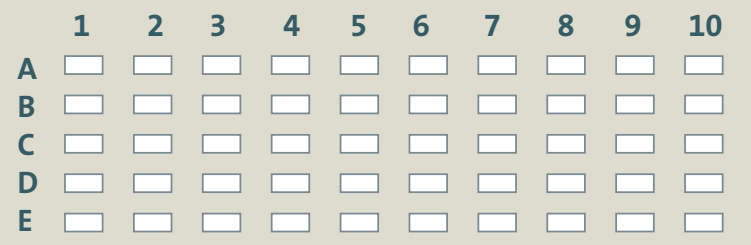

Bitte kreuzen Sie pro Frage je eine Antwort deutlich an.
Und so kommen Sie zu Ihren Punkten:

Teilnahme im Internet: unter www.cme-punkt.de. Dort führen wir für Sie ein elektronisches Punktekonto.

Teilnahme per Brief: Fragebogen ausfüllen und mit einem frankierten Rückumschlag an: Urban \& Vogel Medien und Medizin Verlagsgesellschaft, Redaktion MMW-Fortschritte der Medizin, Abt. Fortbildungszertifikat, Neumarkter Str. 43 81673 München. 